\title{
Equivalence of resolvent and scattering resonances on quantum graphs
}

\author{
Pavel Exner and Jiří Lipovský \\ This paper is dedicated to Jean-Michel Combes on the occasion of his 65th birthday.
}

\begin{abstract}
We discuss resonances for Schrödinger operators on metric graphs which consists of a finite compact part and a finite number of halflines attached to it; the vertex coupling is assumed to be of the $\delta$-type or certain modifications of it. Using exterior complex scaling on the graph we show that the resolvent and scattering resonances coincide in this case.
\end{abstract}

\section{Introduction}

Resonances belong to the class of phenomena which are easy to be understood on a heuristic level but more difficult when we try to study them rigorously. It is not a lack of a precise definition, of course, rather the fact that there are several formal ways in which the problem can be approached. The two oldest and most common concepts are scattering resonances and resolvent resonances. In the first case one inspects the on-shell scattering matrix looking for its sharp changes in some parts of the energy axis suggesting a locally enhanced time delay, while in the second one we inspect analytical continuation of the resolvent to the "unphysical" sheet(s) and look for poles there. The two types of resonances need not be a priori identical, and if fact, there is no reason why they should be, because the latter represent a property of the Hamiltonian $H$ of the system alone, while the scattering refers to a pair $\left(H, H_{0}\right)$ depending thus on the choice of the free operator. Nevertheless, in most systems the scattering resonances referring to a "natural" $H_{0}$ coincide with the resolvent ones, which is a property to be verified in each particular case.

In this paper we are going to address the question of coincidence between scattering and resolvent resonances in noncompact quantum graphs. These systems attracted a lot of attention recently from several reasons, the chief ones among them being that they can be used to model a wide family of semiconductor and other microstructures, and that at the same time they represent a testing ground for investigation of fundamental effects such a quantum chaotic behavior; we refer to Ku04,05 for a review and an extensive bibliography to these problems.

1991 Mathematics Subject Classification. Primary 81U99, 81V99; Secondary 34L25, 34G10.

Key words and phrases. Differential geometry, algebraic geometry.

The research was partially supported by ASCR and MEYS of the Czech Republic within the projects IRP AV0Z10480505, A100480501, and LC06-002. 
From the mathematical point of view quantum graphs represent systems of ODE's coupled by boundary conditions, so the question posed above can be answered in a straightforward way, because the resolvent of a graph Hamiltonian can be constructed by means of Krein's formula. In reality, however, such a head-on approach would be quite laborious because finding the resolvent is not easy unless the graphs in question has a trivial topology. Fortunately, there is a simple way: using a powerful insight of Jean-Michel Combes and collaborators, first formulated in the paper AC71, one can transform the search for poles of the analytically continued resolvent into the spectral problem for a suitable non-selfadjoint operator obtained by an exterior complex scaling of the Hamiltonian. For graphs with $\delta$-coupling at the vertices the goal can be be achieved by combing this method with the well-known duality property E97a. We will first remind some needed notions and illustrate our program on examples, then in Section 3 we will formulate and prove our main result.

\section{Preliminaries}

2.1. Quantum graphs. The configuration space of our model is a graph with a finite number set $\mathcal{V}=\left\{\mathcal{X}_{j}: j \in I\right\}$ of vertices, where $I$ is a corresponding index set, and a set of edges attached to them; the graph is supposed to be metric so the edges are identified with segments of the real axis. In particular, vertices connected to $\mathcal{X}_{j}$ by an edge form the set of neighbors $\mathcal{N}\left(\mathcal{X}_{j}\right)=\left\{\mathcal{X}_{n}: n \in \nu(j) \subset I \backslash\{j\}\right\}$. If necessary we may assume that any two vertices are connected with at most one (finite-length) edge, since otherwise we can add a vertex to each "superfluous" edge. With this convention, each finite-length is uniquely characterized by a pair of vertices, so we can write their set as $\mathcal{L}=\left\{\mathcal{L}_{j n}:\left(\mathcal{X}_{j}, \mathcal{X}_{n}\right) \in I_{\mathcal{L}} \subset I \times I\right\}$. We will assume that a semi-infinite edge is attached to some vertices, denoting the graph and its part with edges of finite lengths as $\Gamma_{e}$ and $\Gamma$, respectively. The graph boundary $\mathcal{B}$ is the set of vertices which have a single neighbor, the interior is $\mathcal{I}=\mathcal{V} \backslash \mathcal{B}$. We denote by $\mathcal{C}$ the set of vertices to which a semi-infinite link is attached, and we introduce $I_{\mathcal{B}}, I_{\mathcal{I}}, I_{\mathcal{C}}$, respectively, as the appropriate index subsets in $I$.

Using the metric structure of our graph we can introduce the state Hilbert space of the problem as the $L^{2}$ space on $\Gamma_{e}$ which is naturally identified with

$$
\mathcal{H}=\bigoplus_{(j, n) \in I_{\mathcal{L}}} L^{2}\left(\left[0, l_{j n}\right]\right) \bigoplus_{j \in I_{\mathcal{C}}} L^{2}([0, \infty))
$$

its elements being written by $\psi=\left\{\psi_{j n}:(j, n) \in I_{\mathcal{L}}, \psi_{j \infty}: j \in I_{\mathcal{C}}\right\}$. The Hamiltonian of the model is a Schrödinger operator on $\Gamma_{e}$ which acts as $-\frac{\mathrm{d}^{2}}{\mathrm{~d} x^{2}}+V_{j n}(x)$ at each edge with a family of potentials $\left\{V_{j n}, V_{j \infty}\right\}$ and appropriate boundary conditions at the vertices. To make things simple we will suppose that $V_{j n} \in L^{\infty}\left(\left[0, l_{j n}\right]\right)$, and moreover, that the motion is free at each external link, $V_{j \infty}=0$. The describe the boundary conditions we need boundary values. Identifying $\mathcal{X}_{j}$ with $x=0$ we introduce $\psi_{j n}=\lim _{x \rightarrow 0+} \psi_{j n}(x)$ and $\psi_{j n}^{\prime}=\lim _{x \rightarrow 0+} \psi_{j n}^{\prime}(x)$, and arrange them as into columns, $\Psi_{j}=\left(\psi_{j n}\left(\mathcal{X}_{j}\right): n \in \nu(j)\right)^{T}$, and similarly for $\Psi_{j}^{\prime}$. The boundary condition at the vertices must ensure that the Schrödinger operator on $\Gamma_{e}$ is self-adjoint; it is well known [KS99 that this happens if

$$
A_{j} \Psi_{j}+B_{j} \Psi_{j}^{\prime}=0, \quad j \in I
$$


where $A_{j}, B_{j}$ are matrices of $d_{j}:=\operatorname{card} \nu(j)$ such that $\left(A_{j}, B_{j}\right)$ has maximal rank $A_{j} B_{j}^{*}$ is self-adjoint at each vertex. Moreover, the non-uniqueness in the above conditions can be removed if one chooses $A_{j}+U_{j}-I, B_{j}=i\left(U_{j}+I\right)$ where $U_{j}$ is a $d \times d$ unitary matrix $\mathbf{H a 0 0}, \mathbf{K S 0 0}$. Hence the family of admissible is characterized by $d_{j}^{2}$ real parameters for each vertex. In this large set there are some distinguished case. One of them is the $\delta$-coupling for which the wave functions are continuous at the vertex, $\psi_{j n}\left(\mathcal{X}_{j}\right)=\psi_{j m}\left(\mathcal{X}_{j}\right)=: \psi_{j}$, and $\sum_{n \in \nu(j)} \psi_{j n}^{\prime}\left(\mathcal{X}_{j}\right)=\alpha_{j} \psi_{j}$. Another interesting case is the $\delta_{s}^{\prime}$-coupling defined by similar conditions with the roles of functions and derivatives interchanged; other examples will be mentioned below.

2.2. Exterior complex scaling. A change of coordinates gives means generally to replace the Hamiltonian by a unitarily equivalent operator. The basic idea of the method mentioned above is to use a family of such transformation, corresponding to scalings in the whole space or in the exterior of a given domain, and to extend it analytically to complex values of the scaling parameter. This typically leads to a non-selfadjoint operator with the essential spectrum rotated; by a suitable parameter choice the resonance poles can be then found as complex eigenvalues of the transformed operator, corresponding to eigenfunctions in the Hilbert space.

For our system a natural idea is to employ a family of transformations which leaves the compact part $\Gamma$ of $\Gamma_{e}$ intact and scales the external semi-infinite edges. Identifying such a link with the halfline $\mathbb{R}^{+}$we consider the scaling transformation $g_{\theta} \rightarrow U_{\theta} g=\mathrm{e}^{\theta / 2} g\left(x \mathrm{e}^{\theta}\right)$ with the parameter $\theta$. Application of $U_{\theta}$ to the Laplacian leads to its multiplication by $\mathrm{e}^{-2 \theta}$, on the halfline one has naturally to transform also the boundary condition. Scaling the external edges, all with same parameter, we replace therefore the original graph Hamiltonian by the operator

$$
H_{\theta}\left(\begin{array}{c}
\left\{g_{j}\right\} \\
\left\{f_{j n}\right\}
\end{array}\right)=\left(\begin{array}{c}
\left\{-\mathrm{e}^{-2 \theta} g_{j}^{\prime \prime}\right\} \\
\left\{-f_{j n}^{\prime \prime}+V_{j n} f_{j n}\right\}
\end{array}\right)
$$

where the upper component corresponds to the external edges, $g_{j}$ being the wave function on the halfline attached to the vertex $\mathcal{X}_{j}$, and $\left\{f_{j n}\right\}$ corresponds similarly to interior edges of the graph. The domain of the transformed operator consists of functions with components $f_{j n} \in W^{2,2}\left(\left[0, l_{j n}\right]\right)$ and $g_{j \theta}=U_{\theta} g_{j}$ with $g_{j} \in W^{2,2}\left(\mathcal{L}_{j \infty}\right)$ satisfying the appropriately transformed boundary conditions. We will be interested in the nontrivial situation when $\theta$ is complex, for instance $\theta=i \vartheta$ with $\vartheta>0$. The essential spectrum of $H_{\theta}$ comes clearly from the external edges and the above formula shows that it is rotates into the lower complex halfplane; for $\vartheta$ large enough one can uncover the poles of $H$ laying on the second sheet.

Before we pass to formulating the general result, let us discuss several examples.

2.3. Example: a line with an appendix. The simplest nontrivial example consist a line, representing to external links, to which a line segment of length $l>0$ is attached at the point $x=0$. Consequently, the Hilbert space is $L^{2}(\mathbb{R}) \oplus L^{2}([0, l])$, its elements can be written as $\psi=\left(\begin{array}{c}g \\ f\end{array}\right)$ where $g, f$ refers to the line and the appendix, respectively, and the Hamiltonian acts as $H \psi=\left(\begin{array}{c}-g^{\prime \prime} \\ -f^{\prime \prime}+V f\end{array}\right)$. To make it self-adjoint we have to choose boundary conditions at the point $x=0$ and the other endpoint of the segment. Let the latter be Dirichlet, $f(l)=0$, and at the vertex we put

$g(0+)=g(0-)=: g(0), \quad f(0)=\beta g(0)+\gamma f^{\prime}(0), \quad g^{\prime}(0+)-g^{\prime}(0-)=\delta g(0)-\beta f^{\prime}(0)$ 
for some $\beta, \gamma, \delta \in \mathbb{R}$ following $\mathbf{E S 9 4}$; it is the most general class of time-reversal Hamiltonians with the line wave function continuous at $x=0$, in particular, the case $\beta=1, \gamma=0$ represents the $\delta$-coupling with $\alpha=\delta$. The scattering problem for this system is easy to solve ES94: denoting by $f_{l}(x)$ the normalized Dirichlet solution to the Schrödinger equation at the appendix, we can express the reflection and transmission amplitudes at energy $k^{2}$ as

$r=\frac{\delta\left[f_{l}(0)-\gamma f_{l}^{\prime}(0)\right]-\beta^{2} f_{l}^{\prime}(0)}{(2 i k-\delta)\left[f_{l}(0)-\gamma f_{l}^{\prime}(0)\right]+\beta^{2} f_{l}^{\prime}(0)}, \quad t=\frac{2 i k\left[f_{l}(0)-\gamma f_{l}^{\prime}(0)\right]}{(2 i k-\delta)\left[f_{l}(0)-\gamma f_{l}^{\prime}(0)\right]+\beta^{2} f_{l}^{\prime}(0)}$.

Scattering resonances coincides with zeros of the denominator; their behavior with respect to the parameters is discussed in $\mathbf{E S 9 4}$. If we suppose for simplicity that $V=0$, then $f_{l}(x)=\sin k(l-x)$ and the condition can be rewritten as

$$
\tan k l=\frac{\beta^{2} k}{2 i k-\delta}-\gamma k
$$

Let us look now at the problem from the complex scaling point of view and put $g_{\theta}(x):=\mathrm{e}^{\theta / 2} g\left(\mathrm{e}^{\theta} x\right)$. The corresponding boundary values are $g_{\theta}(0)=\mathrm{e}^{\theta / 2} g(0)$ and $g_{\theta}^{\prime}(0 \pm)=\mathrm{e}^{3 \theta / 2} g^{\prime}(0 \pm)$. This has to be substituted into the above boundary conditions: the continuity at $x=0$ and the Dirichlet condition at the appendix endpoint do not change, while the other two yield

$$
f(0)=\beta \mathrm{e}^{-\theta / 2} g_{\theta}(0)+\gamma f^{\prime}(0), \mathrm{e}^{-3 \theta / 2}\left[g_{\theta}^{\prime}(0+)-g_{\theta}^{\prime}(0-)\right]=\delta \mathrm{e}^{-\theta / 2}(0) g_{\theta}(0)-\beta f^{\prime}(0) ;
$$

this can be regarded as the boundary conditions which define the non-selfadjoint operator $H_{\theta}$. Assuming again $V=0$, it is easy to solve the corresponding spectral problem. The appendix solution is $f(x)=b \sin k(l-x)$ and its halfline counterparts are the corresponding exponential functions $\mathrm{e}^{\mp i k x \mathrm{e}^{\theta}}$. The first one of the above conditions gives

$$
b=\frac{\beta \mathrm{e}^{-\theta / 2}}{\sin k l+\gamma k \cos k l} g_{\theta}(0)
$$

and substituting from here into the second one we get

$$
\mathrm{e}^{-3 \theta / 2} g_{\theta}(0) 2 i k \mathrm{e}^{\theta}=g_{\theta}(0) \mathrm{e}^{-\theta / 2}\left[\delta-\frac{\beta^{2}(-k \cos k l)}{\sin k l+\gamma k \cos k l}\right] .
$$

It is straightforward to conclude from the last relation that the resolvent resonances for the line with an appendix are determined by the condition (2.2) again.

2.4. Example: a loop with two leads. As the next example let us consider a graph consisting of two internal edges of lengths $l_{1}, l_{2}$, connecting the endpoints of two halflines. Consequently, the Hilbert space is $L^{2}\left(\mathbb{R}^{-}\right) \oplus L^{2}\left(\mathbb{R}^{+}\right) \oplus L^{2}\left(\left[0, l_{1}\right]\right) \oplus$ $L^{2}\left(\left[0, l_{2}\right]\right)$ and states are described by the columns $\psi=(f, g, u, v)^{T}$, with the Hamiltonian acting as $\psi=\left(-f^{\prime \prime},-g^{\prime \prime},-u^{\prime \prime},-v^{\prime \prime}\right)^{T}$; for simplicity we suppose again that the particle is free away of the vertices. The boundary conditions will be now chosen as a $\delta$-coupling at each vertex, i.e. the continuity together with

$$
u^{\prime}(0)+v^{\prime}(0)-f^{\prime}(0)=\alpha f(0), \quad-u^{\prime}\left(l_{1}\right)-v^{\prime}\left(l_{2}\right)+g^{\prime}(0)=\beta g(0) .
$$

The scattering problem is again easy to solve. One uses the above boundary conditions to match the solutions $\mathrm{e}^{i k x}+r \mathrm{e}^{-i k x}$ and $t \mathrm{e}^{i k x}$ for $\mp x>0$ with the linear 
combinations of the exponentials at the internal links; solving the corresponding system of linear equations we get the on-shell reflection and transmission amplitudes

$$
r(k)=\frac{i-\gamma(k)}{i+\gamma(k)}, \quad t(k)=\frac{2 i}{\gamma(k)+i},
$$

where

$$
\gamma(k):=\frac{\left(\frac{1}{\sin k l_{1}}+\frac{1}{\sin k l_{2}}\right)^{2}}{\frac{1}{\tan k l_{1}}+\frac{1}{\tan k l_{2}}+\frac{\beta}{k}-i}-\left(\frac{1}{\tan k l_{1}}+\frac{1}{\tan k l_{2}}\right)-\frac{\alpha}{k} .
$$

In particular, the scattering resonances are now determined by the condition

$$
\gamma(k)+i=0 .
$$

Let us now apply the scaling to both halflines putting $f_{\theta}(x):=\mathrm{e}^{\theta / 2} f\left(x \mathrm{e}^{\theta}\right)$ and $g_{\theta}(x):=\mathrm{e}^{\theta / 2} g\left(x \mathrm{e}^{\theta}\right)$. Calculating the new boundary values we can write down the boundary conditions determining $H_{\theta}$, the continuity at the vertices together with

$$
\begin{gathered}
-\mathrm{e}^{-3 \theta / 2} f_{\theta}^{\prime}(0-)+u^{\prime}(0)+v^{\prime}(0)=\alpha \mathrm{e}^{-\theta / 2} f_{\theta}(0-), \\
\mathrm{e}^{-3 \theta / 2} g_{\theta}^{\prime}(0+)-u^{\prime}\left(l_{1}\right)-v^{\prime}\left(l_{2}\right)=\beta \mathrm{e}^{-\theta / 2} g_{\theta}(0+) .
\end{gathered}
$$

To solve the eigenvalue problem for $H_{\theta}$ one has to match the solutions at the external edges which are $\mathrm{e}^{\mp i k x \mathrm{e}^{\theta}}$ with

$$
u(x)=\frac{f_{\theta}(0-) \sin k\left(l_{1}-x\right)+g_{\theta}(0+) \sin k x}{\sin k l_{1}} \mathrm{e}^{-\theta / 2}
$$

and the analogous solution at the other internal link. This yields the conditions

$$
\begin{aligned}
& k\left[g_{\theta}(0+)\left(\frac{1}{\sin k l_{1}}+\frac{1}{\sin k l_{2}}\right)-f_{\theta}(0-)\left(\frac{1}{\tan k l_{1}}+\frac{1}{\tan k l_{2}}\right)\right]=(\alpha-i k) f_{\theta}(0-), \\
& k\left[g_{\theta}(0+)\left(\frac{1}{\tan k l_{1}}+\frac{1}{\tan k l_{2}}\right)-f_{\theta}(0-)\left(\frac{1}{\sin k l_{1}}+\frac{1}{\sin k l_{2}}\right)\right]=-(\beta-i k) g_{\theta}(0+),
\end{aligned}
$$

which are obviously equivalent to the scattering-resonance equation (2.3).

2.5. Example: a magnetic lasso graph. To show that these consideration extend beyond the pure Schrödinger case, take now a graph consisting of a loop of circumference $l$ to which a halfline is attached and suppose that it is placed into a magnetic field perpendicular to the loop plane. The Hilbert space is thus $L^{2}\left(\mathbb{R}^{+}\right) \bigoplus L^{2}([0, l])$ with elements $\psi=\left(\begin{array}{c}g \\ f\end{array}\right)$ and the Hamiltonian acts as

$$
H \psi=H\left(\begin{array}{l}
g \\
f
\end{array}\right)=\left(\begin{array}{c}
-g^{\prime \prime} \\
-f^{\prime \prime}-2 i A f^{\prime}+A^{2} f
\end{array}\right),
$$

where $A$ is the corresponding vector potential. In fact, the form of the field is not important, what matters is the flux through the loop. The boundary conditions we consider are similar to those of Example 2.3 we suppose that the wave function is continuous on the loop, $f(0)=f(l)$, and furthermore,

$$
f(0)=\alpha^{-1}\left[f^{\prime}(0)-f^{\prime}(l)\right]+\gamma g^{\prime}(0), \quad g(0)=\bar{\gamma}\left[f^{\prime}(0)-f^{\prime}(l)\right]+\tilde{\alpha}^{-1} g^{\prime}(0)
$$

for some $\alpha, \tilde{\alpha} \in \mathbb{R}$ and $\gamma \in \mathbb{C}$; now we do not require time-reversal invariance. Performing the complex scaling on the halfline in the same way as above, the boundary conditions become

$$
\begin{aligned}
f(0) & =\alpha^{-1}\left[f^{\prime}(0)-f^{\prime}(l)\right]+\gamma \mathrm{e}^{-3 \theta / 2} g_{\theta}^{\prime}(0), \\
\mathrm{e}^{-\theta / 2} g_{\theta}(0) & =\bar{\gamma}\left[f^{\prime}(0)-f^{\prime}(l)\right]+\tilde{\alpha}^{-1} \mathrm{e}^{-3 \theta / 2} g_{\theta}^{\prime}(0) .
\end{aligned}
$$


To solve the eigenvalue problem for $H_{\theta}$ we use the following Ansatz: $g_{\theta}(x)=\mathrm{e}^{i k \mathrm{e}^{\theta}}$ and $f(x)=C \mathrm{e}^{-i A x} \sin (k x+\varphi)$ with $\varphi$ given by $\tan \varphi=\sin k l\left(\mathrm{e}^{i A x}-\cos k l\right)$. A straightforward computation yields then the resonance condition

$$
\sin k l-2\left(\frac{k}{\alpha}+\frac{i k^{2}|\gamma|^{2}}{1-i k \tilde{\alpha}^{-1}}\right)(\cos A l-\cos k l)=0,
$$

which is again the same as the following from the scattering on the lasso $\mathbf{E 9 7 b}$.

\section{General graphs}

3.1. Choice of the vertex coupling. In order to extend the observation made in the examples to the class of graphs described in Sec. 2.1 we have first to specify the vertex boundary conditions we will consider, in particular, the way in which the external links are attached to $\Gamma$. For simplicity we suppose that at most one halfline sprouts of each point of the boundary of $\Gamma$. Let us denote the wave function on such a halfline referring to $\mathcal{X}_{j} \in \mathcal{B}$ as $g_{j}$, and those on edges joining $\mathcal{X}_{j}$ with interior vertices as $f_{j n}, j=1, \ldots, m$. To keep things simple we will consider a coupling which generalizes directly the one of Example 2.5 the functions are continuous at the vertex, $f_{j 1}(0)=f_{j 2}(0)=\ldots=f_{j m}(0)=: f_{j}(0)$, and

$$
f_{j}(0)=\alpha_{j}^{-1} \sum_{n=1}^{m} f_{j n}^{\prime}(0)+\gamma_{j} g_{j}^{\prime}(0), \quad g_{j}(0)=\bar{\gamma}_{j} \sum_{n=1}^{m} f_{j n}^{\prime}(0)+\tilde{\alpha}_{j}^{-1} g_{j}^{\prime}(0)
$$

for $\alpha_{j}, \tilde{\alpha}_{j} \in \mathbb{R}$ and $\gamma_{j} \in \mathbb{C}$; it is straightforward to check that it is of the type (2.1). In the interior vertices of $\Gamma$ we suppose a $\delta$ coupling; in the same notation it is continuity again, $f_{j 1}(0)=f_{j 2}(0)=\ldots=f_{j m}(0)=: f_{j}(0)$, and

$$
\sum_{n=1}^{m} f_{j n}^{\prime}(0)=\alpha_{j} f_{j}(0)
$$

for a real $\alpha_{j}$, in general different at different vertices. Finally, if there is a vertex in the boundary of $\Gamma$ to which no external edge is attached, we assume Dirichlet boundary conditions there, $f_{j n}=0$ for $j \in I_{\mathcal{B}} \backslash I_{\mathcal{C}}$.

3.2. A duality. As we have indicated in the introduction, our second main tool will be a duality between Schrödinger equation,

$$
H \psi=k^{2} \psi,
$$

on a graph with a $\delta$-coupling and a certain difference equation. Let us recall it now in more details. To cover both $\Gamma$ and $\Gamma_{e}$ it is useful to formulate the result in a way which allows to describe generalized eigenfunctions at the same time, hence we consider the class $D_{\text {loc }}(H)$ which is the subset in $\bigvee_{(j, n) \in I_{\mathcal{L}}} L^{2}\left(0, \ell_{j n}\right)$ (the direct sum) consisting of the functions which satisfy all the requirements imposed at $\psi \in D(H)$ except the global square integrability.

On the edge $\mathcal{L}_{n j} \equiv\left[0, \ell_{j n}\right]$, with the right endpoint identified to the vertex $\mathcal{X}_{j}$, we denote as $u_{j n}$ and $v_{j n}$ the normalized Dirichlet solutions to $-f^{\prime \prime}+U_{j n} f=k^{2} f$, i.e. those satisfying the boundary conditions

$$
u_{j n}\left(\ell_{j n}\right)=1-\left(u_{j n}\right)^{\prime}\left(\ell_{j n}\right)=0, v_{j n}(0)=1-\left(v_{j n}\right)^{\prime}(0)=0 ;
$$

their Wronskian is $W_{j n}=-v_{j n}\left(\ell_{j n}\right)=u_{j n}(0)$. Since in our case the set $\mathcal{V}$ is finite, the assumptions used in $[\mathbf{E 9 7 a}$ are satisfied and we have the following result. 
Theorem 3.1. Suppose that $\psi \in D_{\text {loc }}(H)$ solves (3.3) for some $k \notin \mathcal{K}$ with $k^{2} \in \mathbb{R}, \operatorname{Im} k \geq 0$. Then the corresponding boundary values satisfy the equation

$$
\sum_{n \in \nu(j) \cap I_{\mathcal{I}}} \frac{\psi_{n}}{W_{j n}}-\left(\sum_{n \in \nu(j)} \frac{\left(v_{j n}\right)^{\prime}\left(\ell_{j n}\right)}{W_{j n}}-\alpha_{j}\right) \psi_{j}=0,
$$

and conversely, any solution $\left\{\psi_{j}: j \in I_{\mathcal{I}}\right\}$ of the system to 3.4 determines a solution of (3.3) by

$$
\begin{array}{r}
\psi_{j n}(x)=\frac{\psi_{n}}{W_{j n}} u_{j n}(x)-\frac{\psi_{j}}{W_{j n}} v_{j n}(x) \quad \text { if } \quad n \in \nu(j) \cap I_{\mathcal{I}}, \\
\psi_{j n}(x)=-\frac{\psi_{j}}{W_{j n}} v_{j n}(x) \quad \text { if } \quad n \in \nu(j) \cap I_{\mathcal{B}} .
\end{array}
$$

When applied to $\Gamma_{e}$ the above result concerns also generalized eigenfunctions. It is useful, however, to specify it for the scattering situation. Let us consider the solutions on the external links $g_{j}(x)=a_{j} \mathrm{e}^{-i k x}+b_{j} \mathrm{e}^{i k x}$ for all $j \in I_{\mathcal{C}}$. The operator $S$ maps the vector of incoming amplitudes $a=\left\{a_{j}\right\}$ into the vector of outgoing amplitudes $b=\left\{b_{j}\right\}$, i.e. $b=S a$. Poles of the scattering matrix are given by the condition $\operatorname{det} S^{-1}=0$; recall that for a nonreal $k$ the matrix ceases to be unitary. Substituting the Ansatz into the boundary conditions (3.1) we obtain for $j \in I_{\mathcal{C}}$

$$
\begin{aligned}
\alpha_{j} \psi_{j} & =\sum_{n=1}^{m} \psi_{j n}^{\prime}(0)+i k \alpha_{j} \gamma_{j}\left(b_{j}-a_{j}\right), \\
\tilde{\alpha}_{j}\left(a_{j}+b_{j}\right) & =\tilde{\alpha}_{j} \bar{\gamma}_{j} \sum_{n=1}^{m} \psi_{j n}^{\prime}(0)+i k\left(b_{j}-a_{j}\right),
\end{aligned}
$$

while for $j \notin I_{\mathcal{C}}$ we have the standard $\delta$-coupling

$$
\alpha_{j} \psi_{j}(j)=\sum_{n \in \nu(j)} \psi_{j n}^{\prime}(j)
$$

Using Theorem 3.1 we can now proceed in the way similar to $\mathbf{E 9 7 \mathbf { b }}$ to obtain the system of equations for $j \in I_{\mathcal{C}}$

$$
\begin{gathered}
\alpha_{j} \psi_{j}=\sum_{n \in \nu(j) \cap I_{\mathcal{I}}}-\frac{\psi_{n}}{W_{j n}}+\sum_{n \in \nu(j)} \frac{v_{j n}^{\prime}\left(l_{j n}\right)}{W_{j n}} \psi_{j}+i k \alpha_{j} \gamma_{j}\left(b_{j}-a_{j}\right) \\
\tilde{\alpha}_{j}\left(b_{j}+a_{j}\right)=\tilde{\alpha}_{j} \bar{\gamma}_{j}\left(\sum_{n \in \nu(j) \cap I_{\mathcal{I}}}-\frac{\psi_{n}}{W_{j n}}+\sum_{n \in \nu(j)} \frac{v_{j n}^{\prime}\left(l_{j n}\right)}{W_{j n}} \psi_{j}\right)+i k\left(b_{j}-a_{j}\right) .
\end{gathered}
$$

On the other hand, for $j \notin I_{\mathcal{C}}$ we have the condition (3.4). We have thus arrived at a system of $N=I+I_{\mathcal{C}}$ equations for $\psi_{j}$ and $b_{j}$ which gives, in particular, the sought S-matrix relating the incoming and outgoing amplitudes.

3.3. Duality for a complex-scaled graph. Let us now perform the exterior complex scaling on $\Gamma_{e}$ which changes the external-edge wave function $g_{j}$ to $g_{j, \theta}(x):=\mathrm{e}^{\theta / 2} g_{j}\left(\mathrm{e}^{\theta} x\right)$; the scaling parameter will be the same for all $j \in I_{\mathcal{C}}$. Our 
aim is to find eigenvalues of the scaled operator $H_{\theta}$, and since there are no potentials on the external links, we know that the solutions there will be of the form $g_{j, \theta}(x)=\mathrm{e}^{i k \mathrm{e}^{\theta} x} g_{j, \theta}(0)$. Substituting from here to (3.1) we get

$f_{j}(0)=\alpha_{j}^{-1} \sum_{n=1}^{m} f_{j n}^{\prime}(0)+i k \gamma_{j} \mathrm{e}^{-\theta / 2} g_{j, \theta}(0), \quad \mathrm{e}^{-\theta / 2} g_{j, \theta}(0)=\bar{\gamma}_{j} \sum_{n=1}^{m} f_{j n}^{\prime}(0)+i k \tilde{\alpha}_{j}^{-1}$,

and eliminating $g_{j, \theta}(0)$ from here we arrive at

$$
f_{j}(0)=\left(\alpha_{j}^{-1}+\frac{i k\left|\gamma_{j}\right|^{2}}{1-i k \tilde{\alpha}_{j}^{-1}}\right) \sum_{n=1}^{m} f_{j n}^{\prime}(0)
$$

In other words, the scaling to replacement of the coupling at the vertex by a new efficient one, non-selfadjoint and energy-dependent, with the parameter

$$
\beta_{j}(k):=\alpha_{j} \frac{1-i k \tilde{\alpha}_{j}^{-1}}{1+i k\left(\left|\gamma_{j}\right|^{2} \alpha_{j}-\tilde{\alpha}_{j}^{-1}\right)},
$$

which applies only to the interior edges meeting at the vertex $\mathcal{X}_{j}$. Now we can repeat step by step the proof of Theorem 3.1 given in $\mathbf{E 9 7 a}$ to find the system of difference equations determining the eigenfunctions of $H_{\theta}$ through their values at the vertices. Those referring to the interior ones, $j \in I_{\mathcal{I}}$, do not change being again given by (3.4). On the other hand, the equations for $j \in I_{\mathcal{C}}$ become

$$
\sum_{n \in \nu(j) \cap I_{\mathcal{I}}} \frac{\psi_{n}}{W_{j n}}-\left(\sum_{n \in \nu(j)} \frac{\left(v_{j n}\right)^{\prime}\left(\ell_{j n}\right)}{W_{j n}}-\beta_{j}(k)\right) \psi_{j}=0
$$

Now we are ready to compare both systems. Substituting from (3.5) into (3.6) we get after a straightforward computation

$$
b_{j}-a_{j}=\frac{\alpha_{j} \bar{\gamma}_{j} \psi_{j}-2 a_{j}}{1+i k\left(a_{j}\left|\gamma_{j}\right|^{2}-\tilde{\alpha}_{j}^{-1}\right)} .
$$

Substituting this into (3.5) again we obtain the system of equations

$$
\sum_{n \in \nu(j) \cap I_{\mathcal{I}}} \frac{\psi_{n}}{W_{j n}}-\left(\sum_{n \in \nu(j)} \frac{\left(v_{j n}\right)^{\prime}\left(l_{j n}\right)}{W_{j n}}-\beta_{j}(k)\right) \psi_{j}=\frac{2 i k \alpha_{j} \gamma_{j} a_{j}}{1+i k\left(\left|\gamma_{j}\right|^{2} \alpha_{j}-\tilde{\alpha}_{j}^{-1}\right)}
$$

and (3.4). As a final step, it is easy to see that the determinants of both system yield the same pole condition.

3.4. The main result. Summarizing the above discussion we are able now to state the claim announced in the introduction about the two resonance sets.

TheOREm 3.2. Let $H$ be a Schrödinger operator on $\Gamma_{e}$ as described in Secs. 2.1 and 3.1, then the families of its resolvent and scattering resonances coincide. 


\section{Concluding remarks}

The situation when the graph loops are pierced by magnetic fluxes the example of which we saw in Sec. 2.5 can treated in the general case also since it reduces to a simple transformation of wave functions on the internal edges [E97a. Furthermore, the duality used in the argument is not restricted to the $\delta$-coupling; in E97a its validity is demonstrated for its $\delta_{s}^{\prime}$-counterpart. On the other hand, it is natural to expect that the equivalence of the two resonance types is valid for any coupling (2.1), however, to prove this claim the present approach needs to be modified.

\section{References}

[AC71] J. Aguilar, J.-M. Combes, A class of analytic perturbations for one-body Schrödinger operators, Commun. Math. Phys. 22 (1971), 269-279.

[E97a] P. Exner, A duality between Schrödinger operators on graphs and certain Jacobi matrices, Ann. Inst. H. Poincaré 66 (1997), 359-371.

[E97b] P. Exner, Magnetoresonances on a lasso graph, Found. Phys. 27 (1997), 171-190.

[EŠ94] P. Exner, E. Šerešová, Appendix resonances on a simple graph, J. Phys. A: Math. Gen. 27 (1994), 8269-8278.

[Ha00] M. Harmer, Hermitian symplectic geometry and extension theory, J. Phys. A: Math. Gen. 33 (2000), 9193-9203.

[KS99] V. Kostrykin, R. Schrader, Kirchhoff's rule for quantum wires, J. Phys. A: Math. Gen. 32 (1999), 595-630.

[KS00] V. Kostrykin, R. Schrader, Kirchhoff's rule for quantum wires. II: The inverse problem with possible applications to quantum computers, Fortschr. Phys. 48 (2000), 703-716.

[Ku04,05] P. Kuchment, Quantum graphs: I. Some basic structures, II. Some spectral properties of quantum and combinatorial graphs, Waves in Random Media 14 (2004), S107-S128; J. Phys. A: Math. Gen. 38 (2005), 269-279.

Doppler Institute for Mathematical Physics and Applied Mathematics, Břehová 7, 11519 Prague, or 25068 Řež near Prague, Czechia

E-mail address: exner@ujf.cas.cz

Institute of Theoretical Physics, Faculty of Mathematics and Physics, Charles

University, V HolešovičKách 2, 18000 Prague, Czechia

E-mail address: JLipovsky@seznam.cz 